Boletín de la Sociedad Geológica Mexicana

Volumen Conmemorativo del Centenario

Grandes Fronteras Tectónicas de México

Toмo LVII, NÚM. 1, 2005, P. 53-63

\title{
La zona de falla Tosco-Abreojos: un sistema lateral derecho activo entre la placa Pacífico y la península de Baja California
}

\author{
François Michaud ${ }^{1, *}$, Thierry Calmus ${ }^{2}$, Marc Sosson ${ }^{1}$, Jean-Yves Royer ${ }^{3}$, Jacques Bourgois ${ }^{1}$, \\ Anne Chabert ${ }^{1}$, Florence Bigot-Cormier ${ }^{1}$, Bill Bandy ${ }^{4}$, Carlos Mortera-Gutiérrez ${ }^{4}$, \\ Jérôme Dyment ${ }^{5}$ \\ ${ }^{1}$ Géosciences Azur, La Darse, BP48, Villefranche/Mer, 06235, Francia. \\ ${ }^{2}$ Instituto de Geología, Universidad Nacional Autónoma de México, Hermosillo, 83000, México. \\ ${ }^{3}$ Centre National de la Recherche Scientifique, Domaines Océaniques, \\ Institut Universitaire Européen de la Mer, Plouzané, F-29280, Francia. \\ ${ }^{4}$ Instituto de Geofísica, Universidad Nacional Autónoma de México, México D.F., 04510, México. \\ ${ }^{5}$ Institut Physique du Globe de Paris, 4 place Jussieu, Paris, 75252, Francia. \\ ${ }^{*}$ micho@obs-vlfr.fr
}

\begin{abstract}
Resumen
A los 12.5 Ma, después del cese de la subducción de la placa Guadalupe debajo de la placa América del Norte, el margen continental actuó como frontera transformante entre las placas Pacífico y América del Norte. La zona de falla Tosco-Abreojos localizada a lo largo de dicho margen fue interpretada como el mejor candidato para acomodar este movimiento transformante antes del Plioceno. A principios del Plioceno la ruptura continental tierra adentro induce la transferencia de la frontera de placas hacia el Golfo de California, y el cese consecuente de la actividad de la zona de falla Tosco-Abreojos como tal. Sin embargo, esta falla presenta una morfología y una actividad sísmica que sugieren la permanencia en la actualidad de un movimiento lateral derecho. Así mismo, la zona de falla Tosco-Abreojos se caracteriza por una alternancia de escarpes y de cuencas asimétricas en las cuales los sedimentos recientes están deformados. Estos resultados son compatibles con los datos cinemáticos (modelos globales y GPS) que indican que el movimiento relativo de la placa Pacífico con respecto a la placa América del Norte está distribuido a lo largo de algunas estructuras, entre ellas la zona de falla Tosco-Abreojos, y el corredor del Golfo de California el cual absorbe la mayor parte de dicho movimiento. La península de Baja California no está totalmente solidaria a la placa Pacífico y aparece como un bloque continental limitado por el Golfo de California al este y la zona de falla Tosco-Abreojos al oeste.
\end{abstract}

Palabras Clave: Falla Tosco-Abreojos, piso oceánico, límite de placas, península de Baja California, placa Pacífico.

Abstract

At 12.5 Ma, after the subduction of the Guadalupe below North America plate has stopped, a right lateral transform motion occurred along the margin, between Pacific and North America plates. The Tosco-Abreojos fault zone, located along the margin of present southern Baja California has been interpreted as the main transform fault between both plates until early Pliocene, when the transform plate boundary has been transferred to the east along the Gulf of California, with the capture of Baja California Peninsula by the Pacific plate. Nevertheless, the morphology and the seismic activity of the Tosco-Abreojos fault zone suggest a present-day right lateral strike-slip motion. The Tosco-Abreojos fault zone 
is characterized by bathymetric scarps and asymmetric basins filled by recent sediments which are deformed. These observations are compatible with cinematic data (GPS and global cinematic models) which suggest that the relative motion of the Pacific plate with respect to the North America plate is partitioned along faults among which the Tosco-Abreojos fault zone, that is not a relict structure, even if the main right-lateral motion is absorbed by the Gulf of California faulted and spreading system. In that way the Baja California Peninsula is considered as an individualized block limited to the west by the Tosco-Abreojos and San Benito fault zones and by the Gulf of California transform boundary to the east.

Key words: Tosco-Abreojos fault, Seafloor morphology, plate boundary, Baja California peninsula, Pacific plate.

\section{Introducción}

La evolución geológica del noroeste de México desde el Mioceno está estrechamente relacionada con los cambios cinemáticos ocurridos entre la placa América del Norte y las placas oceánicas adyacentes. A nivel del Golfo de California, el sistema tectónico actual consiste de un conjunto complejo de fallas transformantes y centros de dispersión maduros e incipientes que une la dorsal del Pacífico Este presente en el desemboque del Golfo de California y el sistema de fallas de San Andrés en California con una longitud total de aproximadamente $1600 \mathrm{~km}$. Con base en los modelos cinemáticos globales, el desplazamiento relativo de la placa Pacífico con respecto a la placa América del Norte varía de $56 \mathrm{~mm} / \mathrm{a}$ (modelo RM2, Relative Motion 2, de Minster y Jordan, 1978) a $50 \mathrm{~mm} / \mathrm{a}$ (modelo NUVEL 1A de DeMets et al., 1990) o $48.8 \pm 1.8 \mathrm{~mm} / \mathrm{a}$ (DeMets, 1995). En la parte norte del Golfo, la correlación entre rocas volcánicas del Mioceno tardío temprano de la región de San Felipe (Baja California) y Sonora a la latitud $29^{\circ} \mathrm{N}$ indican un desplazamiento relativo de $276 \pm 13 \mathrm{~km}$ durante los últimos $6 \mathrm{Ma}$ (Oskin y Stock, 2003), lo cual corresponde a un desplazamiento lateral de $46.0 \pm 0.2 \mathrm{~mm} / \mathrm{a}$. En la parte sur del Golfo de California, las anomalías magnéticas registradas en la cuenca de Alarcón (denominada zona de divergencia Pescadero en Ness et al., 1991, y centro de dispersión Tamayo en Humphreys y Weldon, 1991) indican una velocidad de apertura oceánica entre 58 y $48 \mathrm{~mm} / \mathrm{a}$ durante los últimos 4 millones de años, según los modelo RM2 y NUVEL-1 respectivamente (Humphreys y Weldon, 1991; Ness et al., 1991; Lonsdale, 1995, DeMets y Traylen, 2000). Existe pues una discrepancia entre la velocidad instantánea del desplazamiento relativo entre las placas Pacífico y América del Norte calculada por los modelos cinemáticos y la velocidad calculada a partir de correlaciones geológicas en la región del Golfo de California. Lo anterior significa que parte del movimiento entre ambas placas requiere de la participación de estructuras exteriores al propio Golfo de California.

Así mismo la partición del movimiento entre las placas Pacífico y América del Norte aparece en la diferencia que existe entre la velocidad medida entre las placas Pacífico y América del Norte y la velocidad calculada a partir de los modelos NUVEL-1 y NUVEL-1A (DeMets et al., 1985; DeMets et al., 1990).
La zona de falla Tosco-Abreojos es una zona rectilínea con una longitud de $500 \mathrm{~km}$ localizada a lo largo del margen oeste de Baja California Sur (Figura 1). Junto con el sistema de fallas de San Clemente-San Isidro y San Benito (Krause, 1965) localizado al oeste de la parte norte de Baja California, la zona de falla Tosco-Abreojos forma parte de un sistema de fallas que corre a lo largo de toda la península desde el desemboque del Golfo de California hasta el margen del suroeste de California, en Estados Unidos. La zona de falla Tosco-Abreojos ha sido considerada como activa entre 12 y $5.5 \mathrm{Ma}$ (Spencer y Normark, 1979; Spencer y Normark, 1989), intervalo de tiempo que inicia con el fin de la subducción de la placa Farallón debajo de la placa América del Norte, y termina con la apertura del Golfo de California. Durante este tiempo, se supone que la zona de falla Tosco-Abreojos actuó como falla transformante con desplazamiento lateral derecho, entre los puntos triples de Mendocino y de Rivera que migraron al norte y al sur, respectivamente. Bajo este esquema el conjunto de fallas antes mencionado corresponde al límite de placas durante el Mioceno tardío. Presentamos enseguida un trabajo de revisión de los datos geofísicos que permiten precisar las características de la zona de falla Tosco-Abreojos y su papel en la evolución geodinámica del noroeste de México. La mayor parte de ellos fueron obtenidos durante la campaña geofísica FAMEX cuyos principales resultados fueron publicados en Michaud et al. (2004).

\section{Batimetría}

Los datos batimétricos obtenidos a lo largo de la parte intermedia del margen entre $27^{\circ} \mathrm{N}$ y $24.5^{\circ} \mathrm{N}$ (segmento Abreojos) muestran tres cuencas alargadas (Figura 2) de dirección NW 35-40 (Michaud et al., 2004), las cuales definen la depresión Tosco-Abreojos (Spencer y Normark, 1979). La cuenca sur y la cuenca central tienen una longitud de $45 \mathrm{~km}, 10 \mathrm{~km}$ de ancho y una profundidad máxima de $1800 \mathrm{~m}$. Son cuencas asimétricas, limitadas en su flanco oeste por escarpes empinados con direcciones respectivas

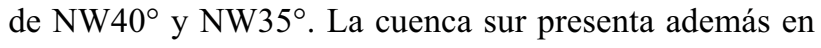
su flanco oeste una estructura de dirección NW $25^{\circ}$ que se prolonga al norte por un cañón de dirección similar y que desemboca en esta misma cuenca.

La cuenca norte tiene una longitud de $70 \mathrm{~km}, 15 \mathrm{~km}$ 


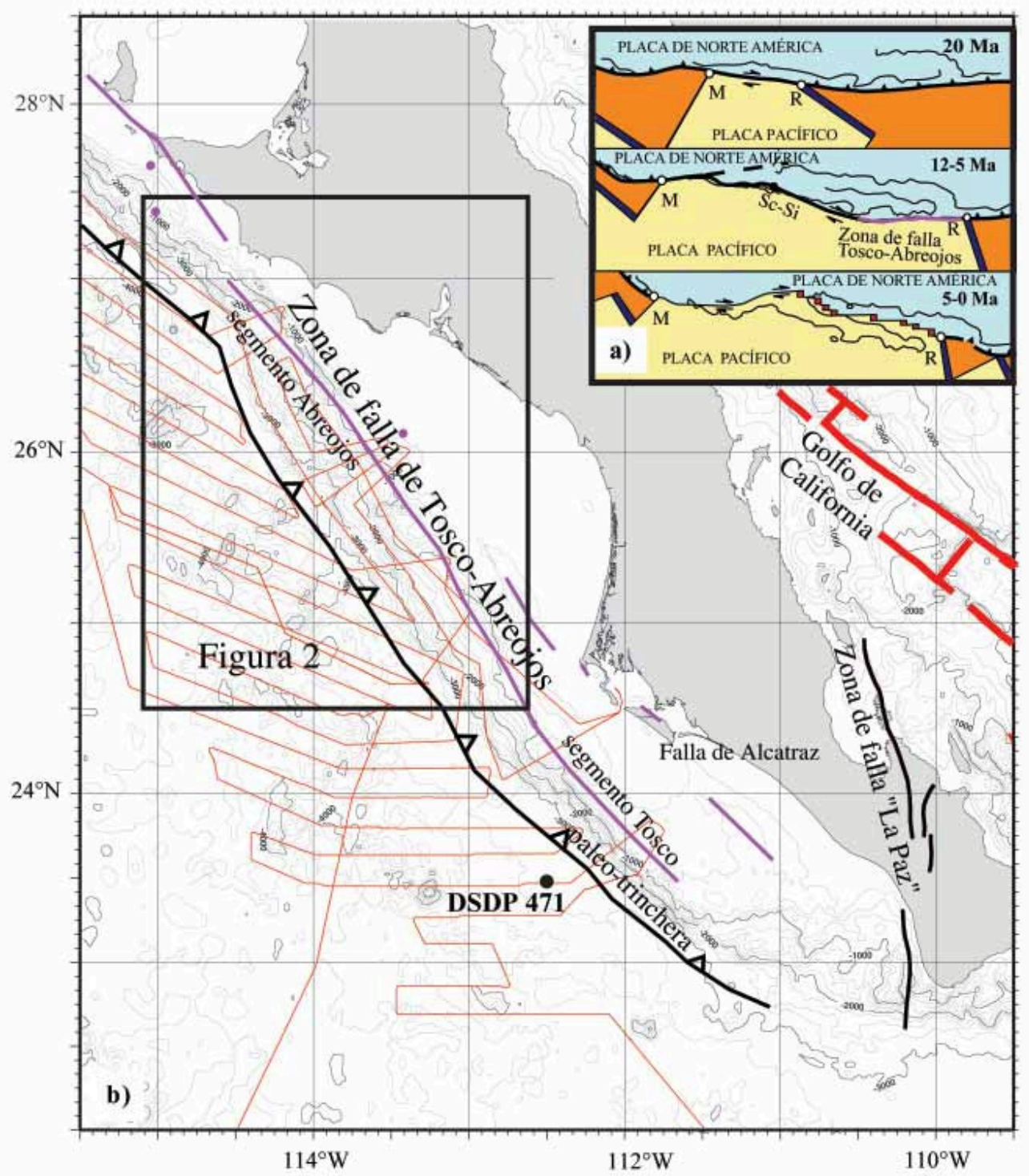

Figura 1. a) Evolución tectónica esquemática del límite entre las placas Pacífico y América del Norte durante los últimos 20 Ma. R: punto triple de Rivera; M: punto triple de Mendocino; Sc-Si: zona de falla San Clemente-San Isidro. b) Carta batimétrica del margen continental y del piso oceánico pacífico (Smith y Sandwell, 1997), así como de una parte del Golfo de California a lo largo de Baja California Sur. A lo largo del margen pacífico de la Península se distingue la zona de falla Tosco-Abreojos y las principales fallas activas de la zona sísmica de Magdalena (Dixon et al., 2000), así como del bloque de los Cabos y de la región de La Paz. La figura muestra también el sistema de fallas transformes y de los puntos de dispersión oceánica Farallón y Carmen en el Golfo de California. El barreno DSDP 471 está localizado (ver discusión en el texto). Las líneas paralelas grises indican las líneas geofísicas del crucero FAMEX (ver texto).

de ancho y una profundidad máxima de $2,600 \mathrm{~m}$. Presenta una asimetría morfológica con un fuerte escarpe de $750 \mathrm{~m}$ hacia el oeste y un escarpe suave con un desnivel de 1,500 $\mathrm{m}$ hacia el este, con una ligera concavidad hacia el oeste y que corresponde a la transición con la parte superior del margen, o plataforma continental (Figura 2). En algunas partes, el piso de la cuenca norte presenta escarpes rectilíneos asociados a pequeños relieves alargados con una altura promedio de $50 \mathrm{~m}$ que indican una actividad tectónica actual (Figura 3). Así mismo, la morfología de la cuenca norte muestra una estructura rectilínea de orientación $\mathrm{NW} 30^{\circ}$, oblicua con respecto a la dirección general de la cuenca, que se sigue desde el sur de la cuenca hasta la parte norte. La orientación $\mathrm{NW} 30^{\circ}$ sugiere que esta estructura representa una falla de desplazamiento lateral actual de segundo orden, de tipo Riedel, con respecto a la dirección principal de movimiento. Dicha estructura se ubica en la misma latitud, $26^{\circ} 50^{\prime} \mathrm{N}$, que el cambio de orientación de la paleotrinchera.

El flanco este de la cuenca central está cortado por una decena de cañones que desembocan de manera abrupta en la cuenca. Este límite muy abrupto de la base de los cañones y la ausencia de abanicos sugieren que la falla que delimita la cuenca al este es activa. 


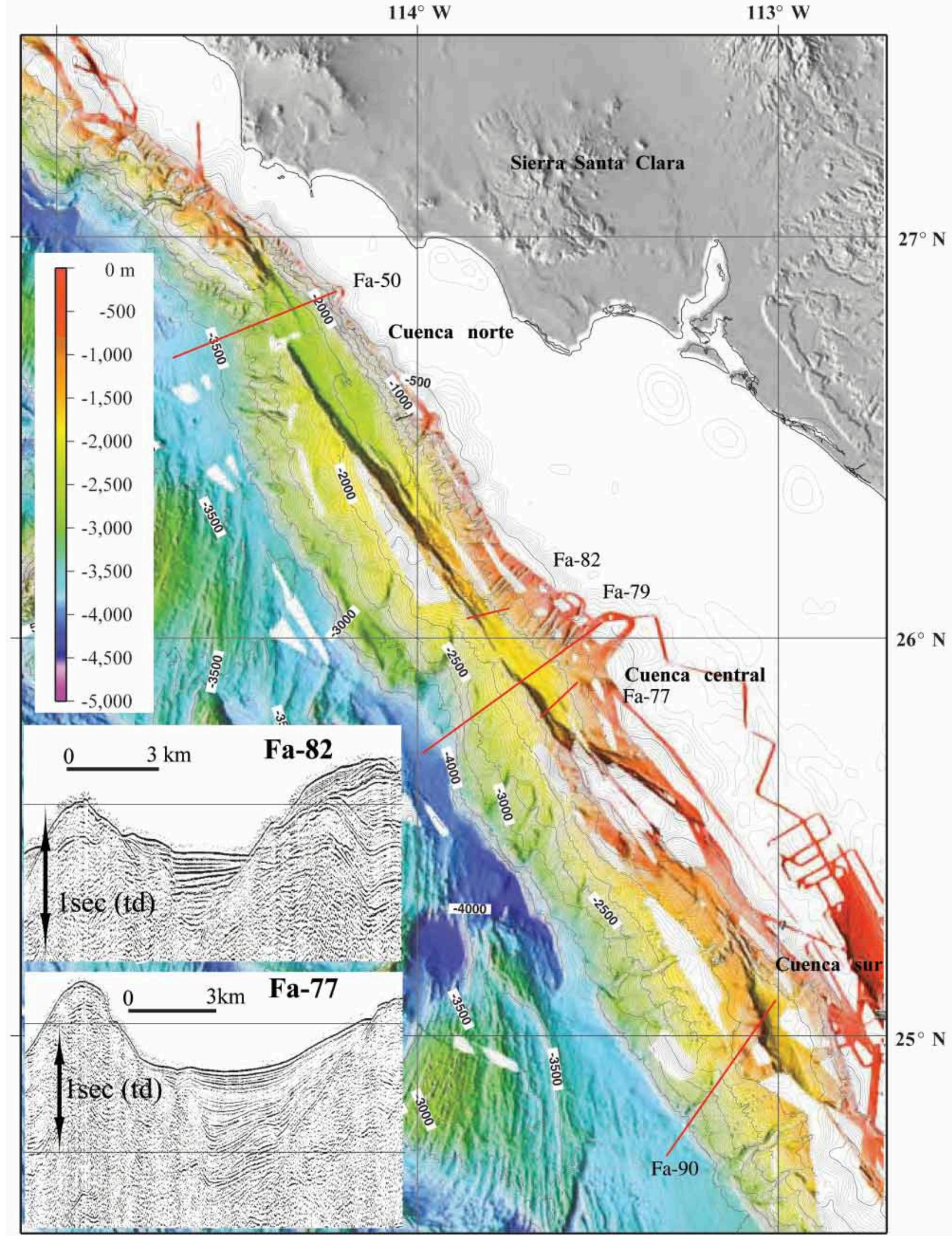

Figura 2. Carta batimétrica del margen de Baja California entre $25^{\circ}$ y $27^{\circ} \mathrm{N}$ adquirida durante el crucero geofísico FAMEX del N/O l'Atalante en 2002. Se distingue en la parte media del margen los escarpes y las cuencas asociadas a la zona de falla Tosco-Abreojos (cuencas norte, central y sur). En la parte inferior izquierda detalles de los perfiles sísmicos Fa-77 y Fa-82 muestran la deformación de los sedimentos asociados a la cuenca central. Los sedimentos de la unidad superior presente en la cuenca central y sobre el alto batimétrico aledaño pertenecen a la unidad A de edad plio-cuaternaria. 


\section{Sísmica de Reflexión}

Los perfiles sísmicos perpendiculares a la zona de falla Tosco-Abreojos entre las latitudes $23^{\circ}$ y $27^{\circ} \mathrm{N}$ confirman las conclusiones obtenidas por los datos batimétricos. Spencer y Normark (1989) indican que la parte sur del sistema de falla Tosco-Abreojos, la zona de falla Tosco, es activa, mientras que la parte norte correspondiente a la depresión de Abreojos es inactiva. Sin embargo, los perfiles sísmicos realizados en 2002 durante la campaña oceanográfica FAMEX (Michaud et al., 2004) confirman que la parte norte del sistema sigue activa al igual que la parte sur (Figura 2). Los escarpes observados en las cartas batimétricas traducen en superficie la presencia de fallas que afectan los sedimentos más recientes como lo muestra el perfil sísmico Fa-50 (Figura 4). En el caso de la cuenca norte, los echados de las fallas este y oeste que limitan la cuenca $30-35^{\circ}$ hacia el oeste, y $40^{\circ}$ hacia el continente respectivamente, sugieren que ambas fallas se intersecan a profundidad, pero la resolución del perfil no permite concluir con certeza (Figura 5). Además, con base a los datos batimétricos, estas fallas se conectan hacia el norte y hacia el sur de la cuenca, definiendo una estructura en flor. Este tipo de estructura se repite a nivel de las cuencas central y sur. El perfil Fa-90 (Figura 6) corta la zona de falla Tosco-Abreojos a la latitud $25^{\circ} \mathrm{N}$, a nivel de la cuenca sur. El relleno sedimentario de la depresión asociada a esta cuenca está afectado por estructuras en flor caracterizadas por la coexistencia de fallas normales e inversas, las cuales dibujan una estructura general sinclinal.

La gran densidad de los perfiles sísmicos realizados durante la campaña FAMEX perpendicularmente al margen oeste de Baja California Sur y el perfil sísmico Fa-113 casi equivalente al perfil sísmico del Leg 63 del programa DSDP que pasa por el sitio 471 (Yeats y Haq, 1981) han permitido intentar hacer una correlación entre las unidades acústicas definidas en el delta submarino de Magdalena y las unidades acústicas encontradas hacia el norte. Enseguida se describen brevemente dichas unidades acústicas barrenadas en el sitio 471. La unidad inferior con un espesor de $440 \mathrm{~m}$ que descansa sobre sedimentos ricos en sulfuros y diabasas, corresponde a las areniscas y turbiditas del Mioceno medio que forman el delta submarino de Magdalena (unidad 4 de Marsaglia, 2004). Las unidades superiores, con un espesor total de $300 \mathrm{~m}$, corresponden principalmente a sedimentos pelágicos que se depositaron a partir de la segunda mitad del Mioceno medio hasta el cuaternario. A la latitud de la cuenca central, en el perfil 79 de la campaña FAMEX (Figura 5), sobre la placa oceánica se distingue unidades equivalentes a las unidades litoestratigráficas del sitio 471 (Yeats y Haq, 1981). La unidad C, que corresponde a la parte inferior de la unidad 4 de Marsaglia (2004), se encuentra en la paleotrinchera y se sigue debajo de la cuña de la placa superior, lo cual indica que la subducción estaba activa todavía en el intervalo de tiempo correspondiente al tiempo de depósito de dicha unidad. Con base en las edades reportadas para las muestras del barreno 471 (Yeats et al.,1981), la unidad C tiene una edad entre 14.5 y 13.5 Ma. La unidad acústica B se distingue de la unidad $\mathrm{C}$ por una mejor reflexión de los sedimentos, equivale a la parte superior de la unidad 4 y a la unidad 3 de Marsaglia (2004) y corresponde al intervalo de tiempo entre $13.5 \mathrm{Ma}$ y aproximadamente 8-7 Ma, con base en la comparación entre la columna estratigráfica propuesta por Marsaglia (2004) y la curva de variación de la tasa de sedimentación en el sitio 471 (Yeats et al., 1981). La unidad B traslapa el contacto entre la placa oceánica y el margen de Baja California. Sin embargo, en el perfil Fa-79, desaparece a nivel de la depresión asociada a la zona de falla ToscoAbreojos. La unidad A es equivalente a las unidades 1 y 2 de Marsaglia (2004). Con base en esta correlación, la unidad A corresponde a un intervalo de tiempo entre 8-7 Ma y el Reciente.

\section{Interpretación Tectónica}

La correlación de las unidades acústicas observadas en el margen con los sedimentos fechados del barreno 471 permite establecer una cronología de los eventos tectónicos que se llevaron a cabo a lo largo del margen oeste de Baja California Sur. La presencia de la unidad C en el plano de subducción indica que la subducción estaba activa todavía durante el Mioceno medio, lo cual coincide con la edad de la reorganización de la cinemática de las placas y del cese de la subducción alrededor de $12.5 \mathrm{Ma}$, correspondiente a la anomalía magnética 5A (Klitgord y Mammerickx, 1982; Mammerickx y Klitgord, 1982; Lonsdale, 1989, 1991). La unidad B, cuya edad se extiende de 13.5 a 8-7 Ma, se encuentra depositada sobre la unidad $\mathrm{C}$ en dominio oceánico y la unidad D, equivalente lateral de la unidad C sobre el margen continental (Figura 4). Sin embargo, los perfiles sísmicos Fa-50 y Fa-79 de la campaña FAMEX (Michaud et al., 2004) muestran que esta unidad está afectada por un fallamiento inverso en la zona de subducción, lo que sugiere que la deformación presentaba todavía una componente de compresión al pie del margen hasta 8-7 Ma. La unidad A corresponde a los sedimentos más jóvenes que 8-7 Ma, que se extienden desde la placa oceánica hasta la trinchera y el margen continental sin discontinuidad aparente. La unidad A está discordante sobre la unidad B porque no está afectada por la deformación en compresión observada en la unidad B a nivel de la trinchera. La unidad A presenta cambios notables de espesor con un engrosamiento asociado a las cuencas. La deformación que afecta esta unidad se concentra exclusivamente a lo largo de la zona de falla Tosco-Abreojos, como lo muestran los perfiles sísmicos Fa-50, Fa-79, Fa-82 y Fa-90 localizados sobre las cuencas norte, central y sur, respectivamente. Sobre estos perfiles, se observa que la unidad A está fallada y plegada en la proximidad de las fallas que limitan las cuencas y adentro de las mismas. La cuenca está limitada por fallas 


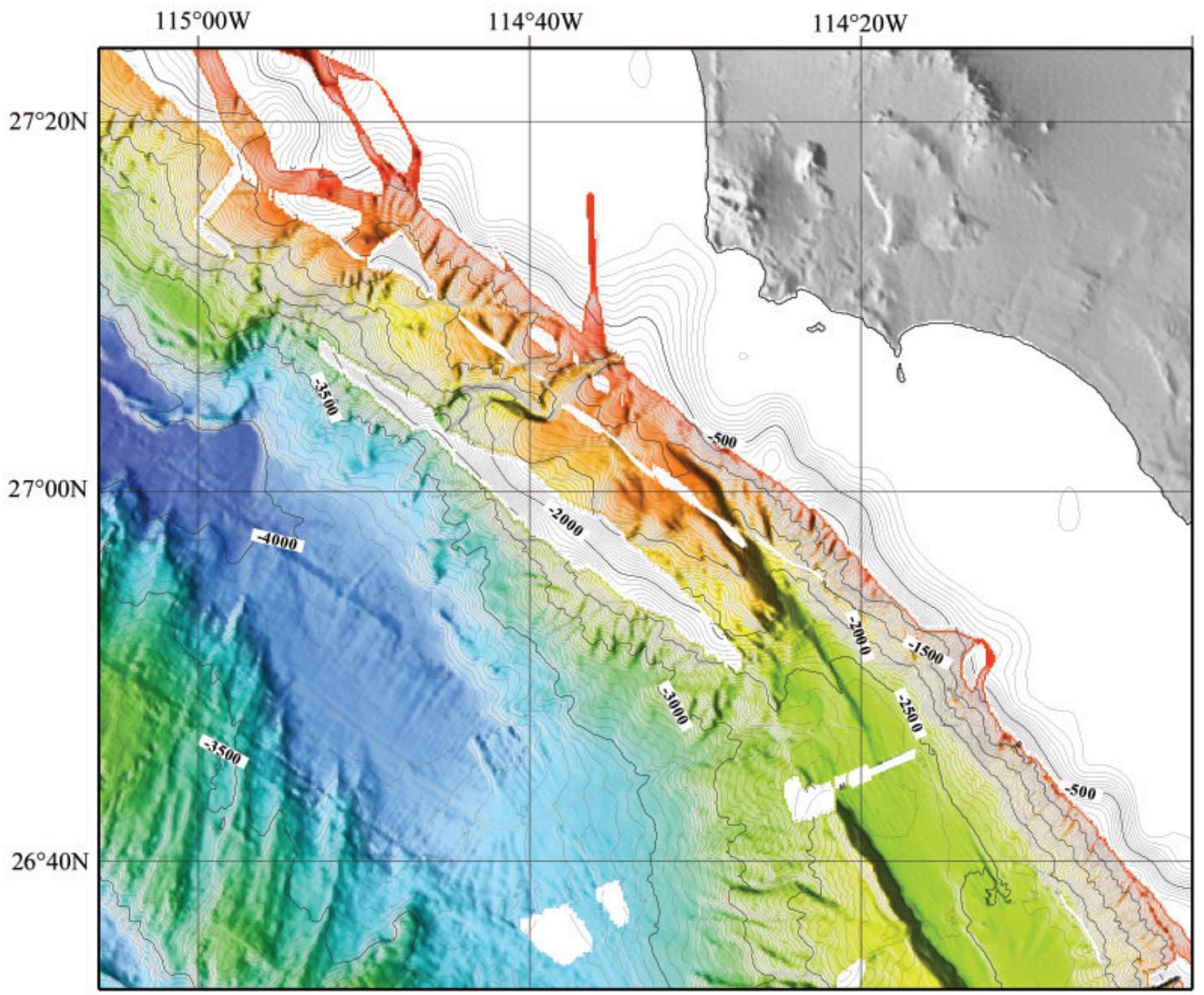

Figura 3. Carta batimétrica a detalle del margen de Baja California Sur entre $26^{\circ} 40$ y $27^{\circ} 20 \mathrm{~N}$. Se observa en la parte sureste de la figura la cuenca norte limitada por un escarpe abrupto al oeste y un escarpe más suave al este. En la parte norte de la cuenca se observa una estructura NW30 $(v e r$ discusión en el texto). En su parte norte, esta estructura se junta con un escarpe de dirección NW50 $0^{\circ}$. Este cambio de dirección corresponde a un giro equivalente de la dirección de la paleotrinchera a la misma latitud. Al norte de la latitud $27^{\circ} \mathrm{N}$, algunos rasgos morfológicos sugieren la actividad reciente o actual de fallas a rumbo derecho como es el cambio brusco de dirección del cañón submarino que adquiere la dirección NW50 $0^{\circ}$ sobre una distancia aproximada de $5 \mathrm{~km}$.

normales y se observa una falla vertical en la parte central de la cuenca central. Al este de la zona de falla ToscoAbreojos, una falla vertical sella la unidad B. El perfil Fa-90 muestra que la cuenca sur está limitada al este por una falla normal de bajo ángulo, y al oeste por una falla normal reactivada en falla inversa. Los sedimentos están plegados y presentan una estructura general sinclinal con complicaciones estructurales en la parte central, en donde se puede observar fallas verticales e inversas. Con base en los datos de sísmica reflexión y de batimetría, se puede concluir que el relleno sedimentario así como la deformación posterior están asociados a la actividad de la zona de falla Tosco-Abreojos.

Con base en las observaciones de sísmica de reflexión y de batimetría de alta resolución, se puede proponer la siguiente evolución estructural del margen durante los últimos 12.5 Ma. Entre 12.5 y 8-7 Ma, la deformación se concentró al pie del margen y posiblemente a lo largo de la zona de falla Tosco-Abreojos. Al movimiento lateral principal está asociada una componente de acortamiento que deforma las unidades C, D y B. Así mismo la componente de acortamiento se manifiesta por la formación de fallas inversas hasta la zona de la paleotrinchera, en donde varios perfiles sísmicos muestran el cabalgamiento de la unidad D sobre la parte inferior de la unidad B. Después de 8-7 Ma, la deformación del margen evoluciona a un régimen transtensional que provoca la formación de las cuencas a lo largo de la zona de falla Tosco-Abreojos. Este cambio de régimen tectónico sobre el margen oeste de Baja California Sur está relacionado con la transferencia de la frontera principal entre las placas Pacífico y América del Norte hacia la zona del futuro Golfo de California a finales del Mioceno tardío y durante el Plioceno. La deformación asociada a la zona de falla Tosco-Abreojos sigue activa durante el Plioceno y la transferencia del límite de placas es progresiva.

La actividad sísmica registrada en la zona sísmica de Magdalena (Dixon et al., 2000) confirma que la zona de 

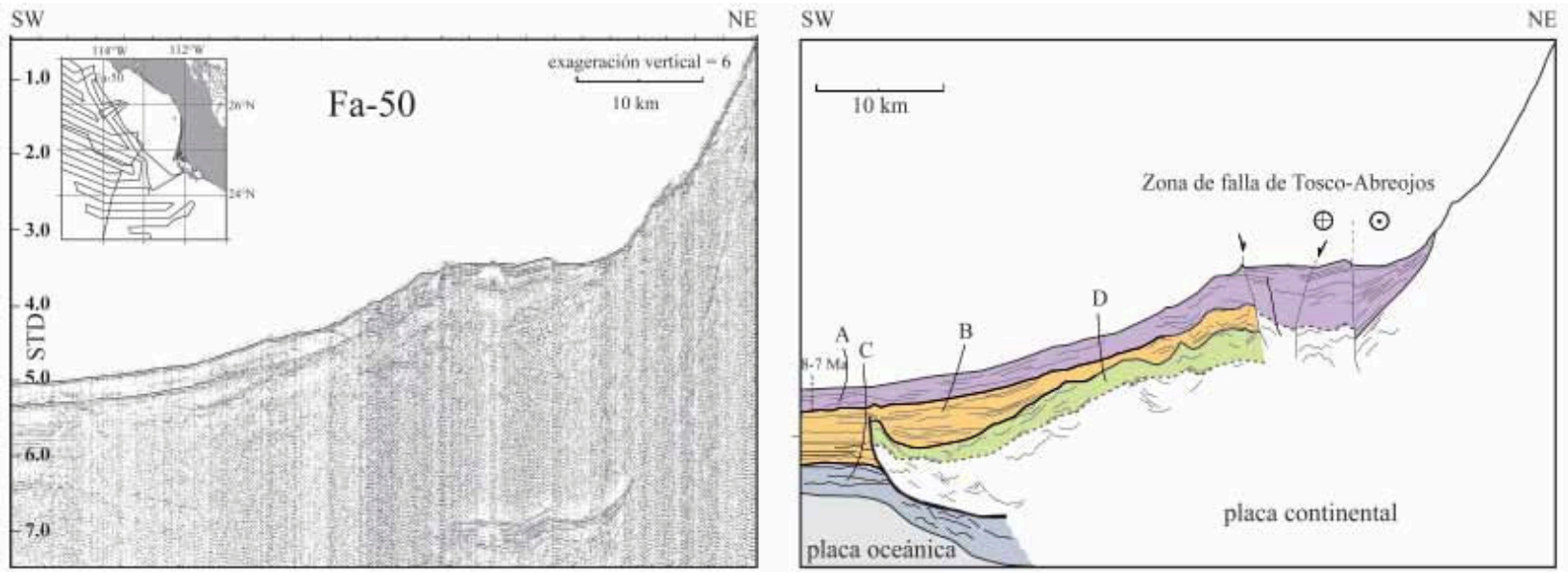

Figura 4. Perfil sísmico Fa-50 (ver texto para la interpretación).

falla Tosco-Abreojos es activa actualmente. Dos sismos de magnitud superior a 5 localizados sobre el trayecto de la falla Tosco, a la latitud de la península de Vizcaíno, y otro sismo de magnitud 4.4 localizado a unos kilómetros al este de la falla Abreojos indican un mecanismo focal derecho con una componente normal a lo largo de rupturas que tienen una dirección de $\mathrm{N} 150^{\circ}+/-5^{\circ}$, compatible con la dirección general de la zona de falla Tosco-Abreojos (Figura 7). De esa manera, la zona de falla Tosco-Abreojos es la estructura más probable para tener el papel de relevo entre el sistema de fallas San Clemente-San Isidro-San Benito alineadas a lo largo de Baja California Norte y la zona símica de Magdalena en donde están reportadas varias fallas activas como la falla de Alcatraz, localizada al norte de la isla Santa Margarita (Yeats y Haq, 1981), la falla Santa Margarita (Normark et al., 1987), la falla Todos Santos y la falla del Carrizal (Ramos-Velázquez, 1998), ambas localizadas en la parte oeste de la región de La Paz-Los Cabos, la falla del cañón submarino de Tinajas, y una probable falla paralela a la costa a una distancia aproximada de 30 $\mathrm{km}$, caracterizada por un sismo de magnitud 5.3 en 1969 (Fletcher y Munguía, 2000).

La misma posición de la zona de falla Tosco-Abreojos, a una distancia corta de la paleotrinchera, sugiere que, al

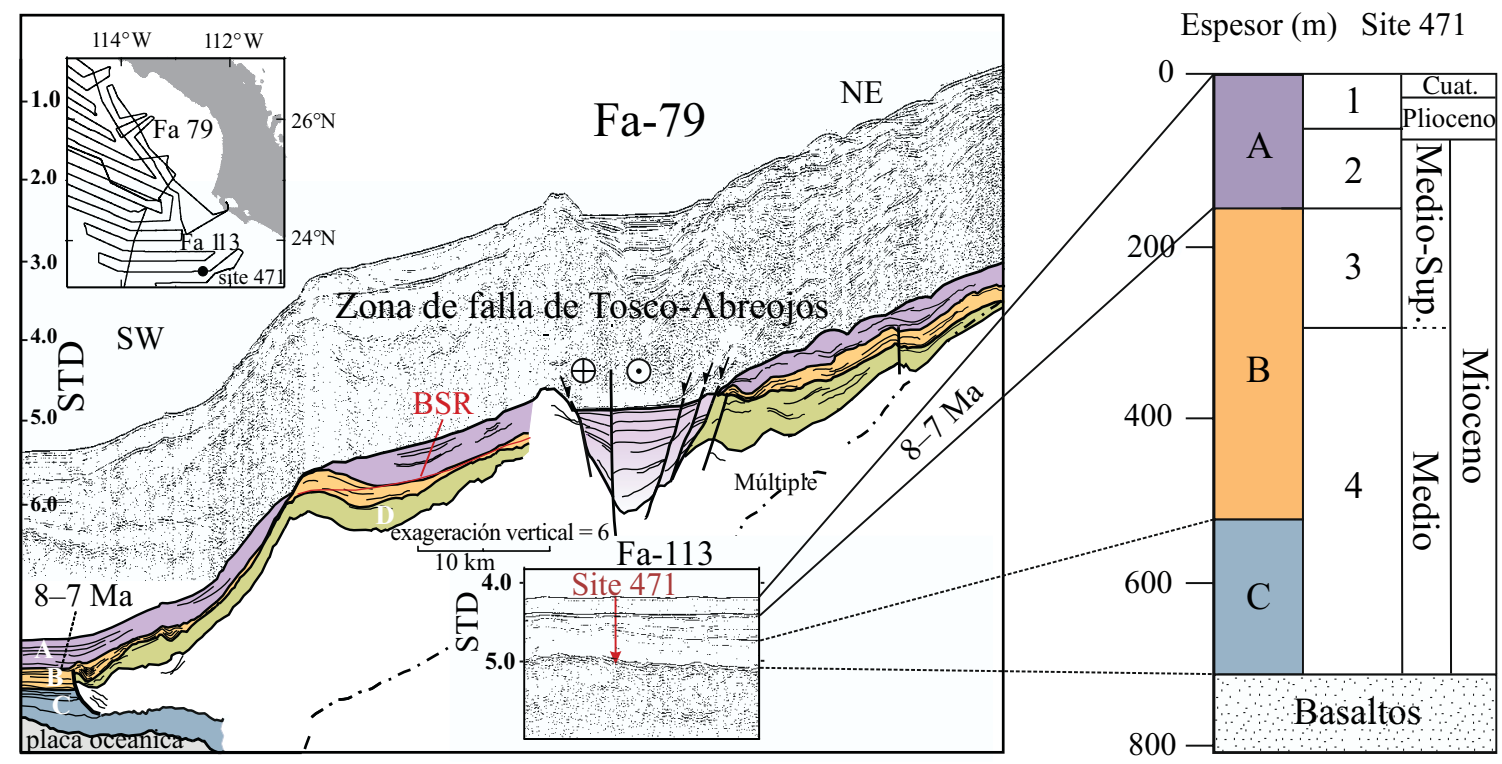

Figura 5. Perfil sísmico Fa-79 e interpretación. Las unidades acústicas son equivalentes a las unidades del perfil sísmico Fa-50 (Figura 4), y correlativas con las unidades acústicas definidas sobre el perfil Fa-113 que pasa por el barreno DSDP 471 (Leg 63). La unidad inferior C está involucrada en el plano de subducción mientras que las unidades B y C se depositaron sobre el margen y la placa oceánica. La unidad B presenta una deformación en compresión ocurrida durante el Mioceno tardío. El margen continental presenta una fuerte deformación a la altura de la zona de falla Tosco-Abreojos (ver texto). La gráfica inferior muestra una columna esquemática de correlación entre las unidades A, B y C (Michaud et al., 2004) y las unidades definidas anteriormente en el sitio 471 (Yeats et al., 1981). Las unidades 1, 2, 3 y 4 están definidas en Marsaglia (2004). 

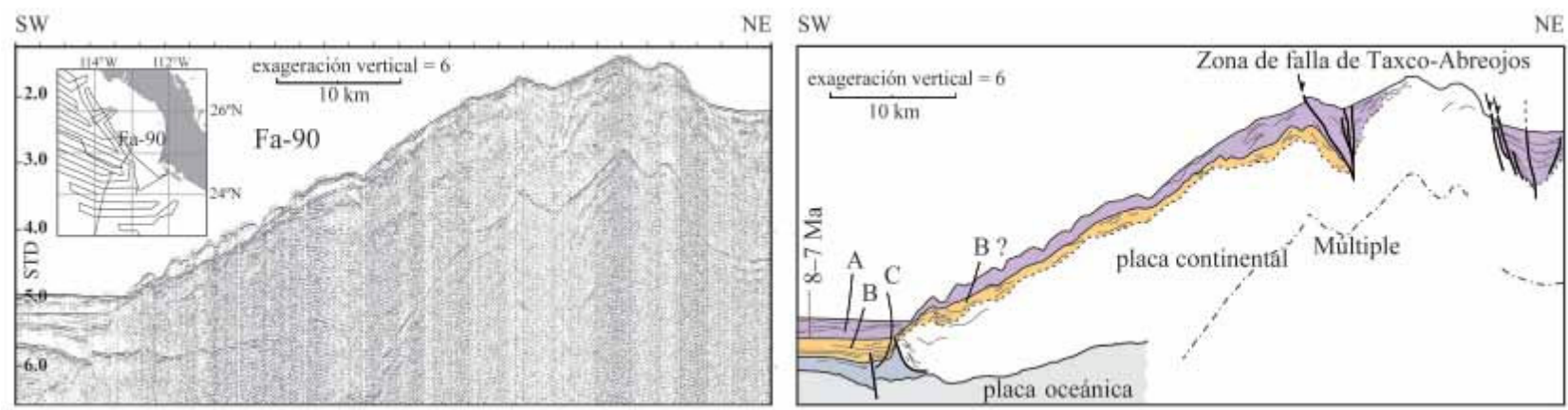

Figura 6. Perfil sísmico Fa-90 e interpretación. El perfil Fa-90, al igual que los perfiles sísmicos Fa-50 y Fa-79, muestra el mismo tipo de estructuras en compresión en la paleo-trinchera y en la zona de falla Tosco-Abreojos. En este perfil la zona de falla presenta dos corredores separados por una alto batimétrico en donde los sedimentos de la unidad A no aparecen debido a una erosión posterior o a una deformación importante no visible por la resolución de la sísmica utilizada.

momento del cierre de la subducción, esta zona de la placa superior correspondía a una zona frágil susceptible de deformarse con más facilidad, debido a: i) la reactivación de una falla lateral izquierda previa relacionada a la subducción oblicua con respecto a la dirección de la trinchera, después del cese de la subducción el movimiento a lo largo de esa falla cambió de izquierdo a derecho para acomodar el nuevo contexto cinemático; ii) la creación de una zona frágil relacionada con una anomalía térmica, provocada por la ruptura de la placa oceánica en subducción debajo de la margen adelgazada de la placa superior.

\section{Discusión}

La mayor parte del movimiento relativo entre las placas Pacífico y América del Norte es absorbido en la región del Golfo de California. Las anomalías magnéticas del piso oceánico de los centros de dispersión de la parte sur del Golfo y de su desemboque registraron una aceleración de 10 a $15 \%$ de la divergencia entre Baja California y la placa América del Norte (DeMets, 1995), pasando de una velocidad de $44.8 \mathrm{~mm} / \mathrm{a}$ a los $3.58 \mathrm{Ma}$ (anomalía 2An.3) a $49.8 \mathrm{~mm} / \mathrm{a}$ a $0.78 \mathrm{Ma}$ (anomalía $1 \mathrm{n}$ ). Sin embargo, la velocidad del movimiento de la placa Pacífico con respecto a la placa América del Norte es constante desde 3.16 Ma, lo que significa que parte del movimiento se acomoda a lo largo de estructuras exteriores ubicadas al oeste del Golfo de California, como son la zona de falla Tosco-Abreojos y las fallas de la zona sísmica de Magdalena (Dixon et al., 2000; Fletcher y Munguía, 2000). El hecho que la velocidad de dicho movimiento es constante durante los últimos 3.16 millones de años, y que la velocidad de desplazamiento de Baja California con respecto a la misma placa América del Norte se incrementó hasta los $0.78 \mathrm{Ma}$, implica que la velocidad de desplazamiento a lo largo de la zona de falla Tosco-Abreojos disminuyó durante el mismo intervalo de tiempo, y que Baja California se desplazó con respecto a la placa Pacífico antes de 0.78 Ma.
Sin embargo, las velocidades determinadas por GPS para algunos sitios de Baja California con respecto a América del Norte siguen inferiores a la velocidad de la placa Pacífico con respecto a la misma placa América del Norte (Dixon et al., 2000). Es el caso por ejemplo del sitio CONC $\left(26.62^{\circ} \mathrm{N}, 111.81^{\circ} \mathrm{W}\right)$ localizado en Bahía Concepción sobre la costa este de Baja California Sur (Figura 8). El sitio CONC se desplaza con una velocidad de $43.6 \pm 2.2 \mathrm{~mm} / \mathrm{a}$ con respecto a la placa América del
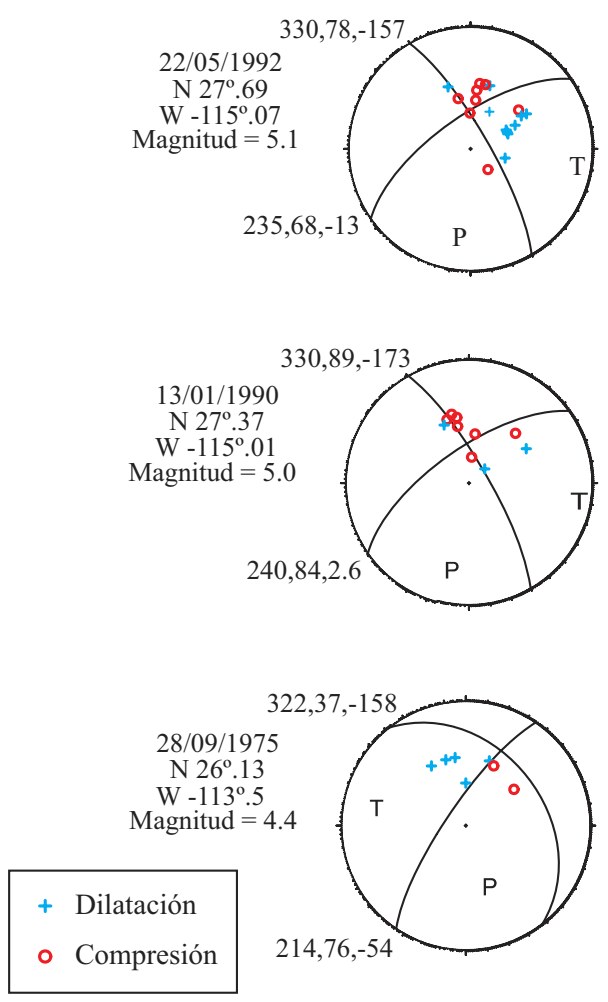

Figura 7. Resolución de los mecanismos focales de 3 sismos localizados sobre el margen oeste de Baja California Sur, cercanos a la zona de falla Tosco-Abreojos. Los tres sismos presentan un mecanismo focal lateral derecho con una componente normal, compatible con los datos cinemáticos y el análisis morfo-estructural (Michaud et al., 2004). 


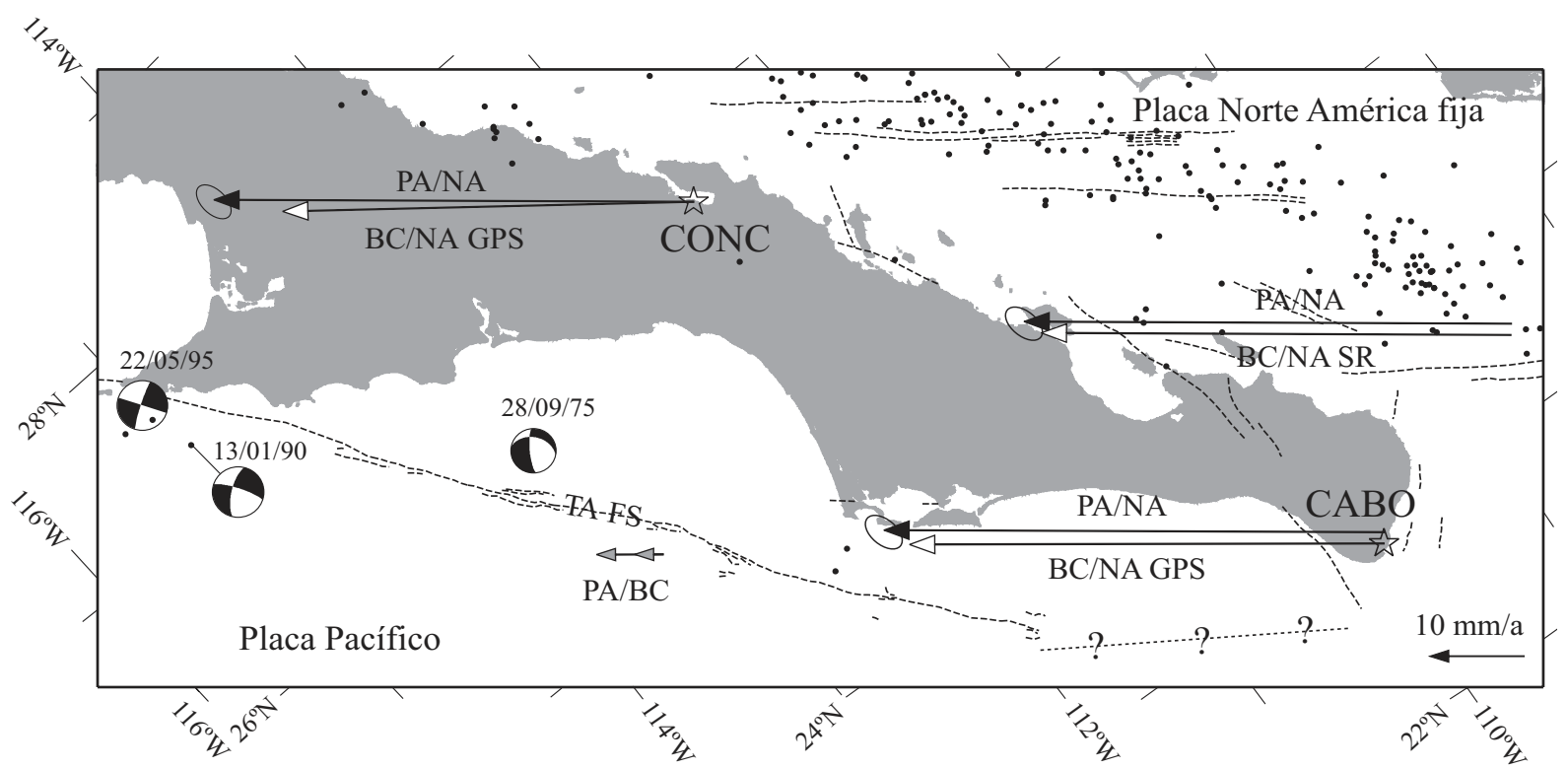

Figura 8. Predicción y observaciones de la cinemática de Baja California Sur con respecto a la placa América del Norte. Las flechas negras indican el desplazamiento de la placa Pacífico con respecto a la placa América del Norte (Argus y Gordon, 2001). Las flechas blancas muestran el desplazamiento de Baja California basado sobre las mediciones de GPS realizadas en los sitios CONC, Bahía Concepción y CABO, Cabo San Lucas (Dixon et al., 2000) y sobre la medición de las anomalías magnéticas a nivel de la dorsal de Alarcón en el sur del Golfo de California para los últimos 0.78 Ma (DeMets, 1995). Las velocidades máxima, $7 \mathrm{~mm} / \mathrm{a}$, y mínima, $3 \mathrm{~mm} / \mathrm{a}$, de la placa Pacífico con respecto a Baja California Sur, son indicadas por las flechas grises. Corresponden a la diferencia entre la cantidad de desplazamiento relativo entre las placas Pacífico y América del Norte y el desplazamiento relativo de Baja California Sur con respecto a América del Norte y son compatibles con el movimiento lateral derecho y la componente normal observados a lo largo de la zona de falla Tosco-Abreojos y con los mecanismos focales de sismos (ver Figura 7). Los epicentros (puntos negros) provienen del catalogo del Centro Nacional de Información Sismológica del Servicio Geológico de Estados Unidos (USGS). MSZ: Zona Sísmica de Magdalena. Proyección Mercator oblicua con respecto al punto de rotación de la placa Pacífico con respecto a la placa América del Norte.

Norte que es inferior a la velocidad predicha de $50.7 \mathrm{~mm} / \mathrm{a}$ de la placa Pacífico con respecto a la placa América del Norte (Dixon et al., 2000; Argus y Gordon, 2001). Dixon et al. (2000) señalan que la diferencia entre la velocidad observada y la velocidad predicha se debe a la acumulación de deformación elástica a lo largo de posibles fallas activas cercanas al sitio de medición, $\mathrm{o}$ a desplazamientos laterales a lo largo de fallas submarinas localizadas en el margen oeste de Baja California. El acimut del vector de desplazamiento del sitio CONC cambia ligeramente según los estudios: Dixon et al. (2000) proponen un acimut de $308.4^{\circ} \pm 3.0$, y Antonelis et al. (1999) proponen un acimut de $303.9^{\circ} \pm 3.2$. Ambos resultados son ligeramente oblicuos con respecto a la dirección de la zona de falla Tosco-Abreojos ( $15^{\circ}$ en el primer caso). Si consideramos la hipótesis que no existen fallas activas entre el sitio CONC y la zona de falla Tosco-Abreojos, el desplazamiento actual a lo largo de la zona de falla Tosco-Abreojos corresponde a la diferencia observada entre la velocidad predicha y la velocidad observada en el sitio CONC, es decir $7.1 \mathrm{~mm} / \mathrm{a}$. Debido a la oblicuidad entre el vector desplazamiento y la dirección de la zona de falla Tosco-Abreojos el desplazamiento presenta una componente lateral derecha de $6.9 \mathrm{~mm} / \mathrm{a}$ y un componente extensional de $1.8 \mathrm{~mm} / \mathrm{a}$. Este resultado es compatible con las observaciones batimétricas y estructurales realizadas sobre la falla. Sin embargo estos valores son máximos y podrían ser menores en la medida que exista fallas activas entre el sitio CONC y la zona de falla Tosco-Abreojos que absorben parte del movimiento de forma elástica, por ejemplo las fallas asociadas al escarpe que limita la Provincia Extensional del Golfo, o las fallas localizadas en la parte occidental de la península a lo largo de las cuales se emplazaron rocas volcánicas andesíticas durante el Plioceno tardío y el Cuaternario. La estabilidad del movimiento relativo de la placa Pacífico con respecto a la placa América del Norte desde 3.16 Ma y la aceleración reciente de la acreción oceánica en el Golfo de California indica que las tasas de desplazamiento lateral y de extensión fueron aún más importantes antes de $1 \mathrm{Ma}$ del orden de 12 y $3 \mathrm{~mm} / \mathrm{a}$, respectivamente. Para el periodo anterior a 3.16 Ma, el modelo de Atwater y Stock (1998) considera un movimiento más rápido entre las placas Pacífico y América del Norte, superior a $56 \mathrm{~mm} / \mathrm{a}$ entre 3.2 y 10.9 $\mathrm{Ma}$, establecido en el sitio CONC. Considerando que el movimiento en el Golfo de California era equivalente o inferior a la tasa inicial de acreción oceánica de $45 \mathrm{~mm} / \mathrm{a}$ para la anomalía 2A (DeMets, 1995), la velocidad del movimiento relativo entre Baja California y la placa Pacífico debe haber estado por lo menos igual y probablemente más importante que la velocidad observada actualmente. La historia cinemática de la región sugiere que la falla Tosco-Abreojos permaneció activa durante los últimos 10 
millones de años con un decrecimiento progresivo de la tasa de desplazamiento lateral.

\section{Conclusiones}

Los levantamientos de batimetría y de sísmica reflexión recientes permiten precisar la evolución tectónica de la zona de falla Tosco-Abreojos. Actualmente es una falla activa a lo largo de la cual ocurre un desplazamiento lateral derecho con una componente transtensional que es congruente con una predicción cinemática de 2.9 a $6.9 \mathrm{~mm} / \mathrm{a}$. Los modelos cinemáticos sugieren que el movimiento de la placa Pacífico con respecto a la placa América del Norte no está totalmente acumulado en el Golfo de California, sino que está distribuido (DeMets, 1995; DeMets y Dixon, 1999; Dixon et al., 2000; Fletcher y Munguía, 2000). Un porcentaje significativo del movimiento se distribuye de manera discreta a lo largo de una zona ancha (Mann et al., 1983) cuyo límite más occidental es la zona de falla ToscoAbreojos, y cuyo límite oriental se ubica probablemente a lo largo de la costa de Sonora. La zona de falla ToscoAbreojos registra actualmente un desplazamiento lateral derecho entre la Península de Baja California y la placa Pacífico, lo cual permite definir un bloque Baja California limitado al oeste por la zona de falla Tosco-Abreojos y al este por el sistema transformante del Golfo de California, conformado por una sucesión de fallas laterales y cuencas de tipo pull-apart, algunas siendo centros de dispersión oceánica en la parte sur del Golfo. Hacia el norte, la zona de falla Tosco-Abreojos se conecta con el sistema de fallas San Isidro-San Clemente que forman un enjambre de fallas submarinas a lo largo del margen de los estados de Baja California, México y de California, Estados Unidos de América (Legg et al., 1991; Humphreys y Weldon, 1991). La prolongación hacia el sur de la zona de falla ToscoAbreojos es más problemática, pero, con base en los datos disponibles, se pueden seguir escarpes rectilíneos paralelos a la zona de falla Tosco-Abreojos hacia el sur, en donde varias fallas sustituyen a la zona de falla Tosco-Abreojos, tales como la falla Alcatraz (Yeats y Haq, 1981), la falla Santa Margarita (Normark et al., 1987), y el sistema de fallas principalmente normales del bloque de Los Cabos como la falla Todos Santos, la falla del Carrizal (Fletcher y Munguía, 2000), las cuales a su vez representan un sistema de relevo con la zona de falla de Tamayo en la parte sur del Golfo de California.

\section{Agradecimientos}

Agradecemos a la tripulación y al equipo técnico del N/O L'Atalante (Genavir/Ifremer), a Luc Beaufort, Jefe de la Misión MONA a bordo del N/O MARION DUFRESNE II (Genavir/Ifremer), así como a Lex VanGreen, Jefe de la Misión OXMZ01MV swl N/O MELVILLE y a Barry
Eakins. Este trabajo se ha beneficiado del apoyo del Instituto Nacional de Ciencias del Universo del Centro Nacional de Investigación Científica de Francia. Los autores agradecen a Susana A. Alaniz Alvarez y a José Rosas Elguera por las sugerencias que permitieron enriquecer el presente trabajo.

\section{Referencias Bibliográficas}

Antonelis, K., Johnson, D. J., Miller, M. M., Palmer, R., 1999, GPS determination of current Pacific-North American plate motion, Geology, 27, 299-302.

Argus, D. F., Gordon, R. G., 2001, Present tectonic motion across coastal ranges and San Andreas fault system in central California, Geological Society of America Bulletin, 113, 1580-1592.

Atwater, T., Stock, J., 1998, Pacific-North America Plate Tectonics of the Neogene Southwestern United States: An Update, International Geology Review, 40, 375-402.

DeMets, C., 1995, A reappraisal of seafloor spreading lineations in the Gulf of California: Implications for the transfer of Baja California to the Pacific plate and estimates of Pacific-North America motion, Geophysical Research Letters, 22, 3545-3548.

DeMets, C., Dixon, T.H., 1999, New kinematic models for Pacific-North America motion from 3 Ma to present, I: Evidence for steady motion and biases in the NUVEL-1A model, Geophysical Research Letters, 26, 1921-1924.

DeMets, C., Gordon, R. G., Stein, S., Argus, D. F., Engeln, J., Lundgren, P., Quible, D. G., Stein, C., Weinstein, S. A., Wiens, D. A., Woods, D. F., 1985, NUVEL-1: A new global plate motion data set and model, Eos Transactions AGU, 66, 368-369.

DeMets, C., Gordon, R. G., Argus, D. F., Stein, S 1990, Current plate motions, Geophysical Journal International, 101, 425-478.

DeMets, C., Traylen S., 2000, Motion of the Rivera plate since $10 \mathrm{Ma}$ relative to the Pacific and North American plates and the mantle, Tectonophysics, 318, 1-4, 119-160.

Dixon, T., Farina, F., DeMets, C., Suarez-Vidal, F., Fletcher, J., MarquezAzua, B., Miller, M., Sanchez, O., Umhoefer, P., 2000, New kinematic models for Pacific-North America motion from 3 Ma to Present II: Evidence for a "Baja California shear zone", Geophysical Research Letters, 27, 3961-3964.

Fletcher, J.M., Munguía, L., 2000, Active continental rifting in southern Baja California, Mexico: Implications for plate motion partitioning and the transition to seafloor spreading in the Gulf of California, Tectonics, 19, 6, 1107-1123.

Humphreys, E. D., Weldon II, R. J., 1991, Kinematic constraints on the rifting of Baja California, en Dauphin, J. P., y Simoneit, B. R. T., (eds.), The Gulf and Peninsular Province of the Californias, American Association of Petroleum Geologists, Memoir 47, 217- 229.

Klitgord, K. D., Mammerickx, J., 1982, Northern East Pacific Rise: Magnetic anomaly and bathymetric framework, Journal of Geophysical Research, 87, 6725-6750.

Krause, D. C., 1965, Tectonics, bathymetry, and geomagnetism of the southern continental bordeland west of Baja California, Mexico, Geological Society of America Bulletin, 76, 617-650.

Legg, M.R., Wong, O., V. Suarez, 1991, Geologic structure and Tectonics of the Inner Continental Bordeland of the Northern Baja California, en Dauphin, J. P., y Simoneit, B., (eds.), The Gulf and Peninsular Province of the Californias, AAPG Memoir, 47, 145- 196.

Lonsdale, P., 1989, Geology and tectonic history of the Gulf of California, en Winterer, E. L., Hussong, D. M., y Decker, R. W., (eds.), The Eastern Pacific Ocean and Hawaii, The Geology of North America, Geological American Society, N., 499- 521.

Lonsdale, P., 1991, Structural patterns of the Pacific floor offshore of peninsular California, en Dauphin, J. P., y Simoneit, B.R.T., (eds.), The Gulf and Peninsular Province of the Californias, American Association of Petroleum Geologists, Memoir 47, 87- 125. 
Lonsdale, P., 1995, Segmentation y disruption of the East Pacific Rise in the mouth of Gulf of California, Marine Geophysical Researches, 17, 323-359.

Mammerickx, J., Klitgord, K. D., 1982, Northern East Pacific Rise: Evolution from 25 m.y. B.P. to the Present, Journal of Geophysical Research, 87, 6751-6759.

Mann, P., Hempton, M. R., Bradley, D. C., Burke, K., 1983, Development of pull-apart basins, Journal of Geology, 91, 529-554

Marsaglia, K. M., 2004, Sandstone detrital modes support Magdalena Fan displacement from the mouth of the Gulf of California, Geology, 32, 45-48.

Michaud, F., Sosson, M., Royer, J. Y., Chabert, A., Bourgois, J., Calmus, T., Mortera, C., Bigot-Cormier, F., Bandy, W., Dyment, J., Pontoise, B., Sichler, B., 2004, Motion partitioning between the Pacific plate, Baja California and the North America plate: The Tosco-Abreojos fault revisited, Geophysical Research Letters, 31, L08604.

Minster, J. B., Jordan, T. H., 1978, Present-day plate motions, Journal of Geophysical Research, 83, 5331-5354.

Ness, G. E., Lyle, M. E., Couch, R. W., 1991, Marine magnetic anomalies and oceanic crustal isochrones of the Gulf and Peninsular Province of the Californias, en Dauphin, J. P., y Simoneit, B. R. T., (eds.), The Gulf and Peninsular Province of the Californias, American Association of Petroleum Geologists, Memoir 47, 47- 69.

Normark, W., Spencer, J., Ingle, J., 1987, Geology and Neogene history of the Pacific margin of Baja California Sur, en Geology and Resource Potential of the Continental Margin of Western North America, Earth Science Series, 6, Scholl, D., Grantz, A, y Vedder, J., (eds). Circum Pacific Counsel Energy Min. Resources, Houston, Tx, 449-472.

Oskin, M., Stock, J., 2003, Pacific-North America plate motion and opening of the Upper Delfín basin, northern Gulf of California, Mexico, Geological Society America Bulletin, 115, 1173-1190.

Ramos-Velázquez, E., 1998, Características de la deformación en las rocas cristalinas cretácicas del este sureste de la ciudad de La Paz, BCS, México, Tesis de Maestría, Centro de Investigación Científica y de Educación Superior de Ensenada, Ensenada, México, 122 pp.
Smith, W. H. F., Sandwell, D. T., 1997, Global sea floor topography from satellite altimetry and ship depth soundings, Science, 277, 1957-1962.

Spencer, J. E., Norwark, W., 1979, Tosco-Abreojos fault zone: A Neogene transform plate boundary within the Pacific margin of southern Baja California, Mexico, Geology, 7, 554-557.

Spencer J.E., W. Normark, 1989, Neogene plate-tetonic evolution of the Baja California Sur continental margin and the southern Gulf of California, Mexico, en Winterer, E.L., Hussong, D.M., y Decker, R., (eds.), The Eastern Pacific Ocean and Hawaii: Boulder, Colorado, The Geology of North America, Geological Society of America, N, 489-497.

Yeats et al., 1981, Site 471: Offshore Magdalena Bay, en Orlofsky, S. (ed.), Initial Reports of the Deep Sea Drilling Project, 63: Washington (U.S. Government Printing Office), 269-323.

Yeats, R.S., y Haq, B.U., 1981, Deep-Sea drilling off the Californias: Implications of Leg 63, en Orlofsky, S. (ed.), Initial Reports of the Deep Sea Drilling Project, 63: Washington (U.S. Government Printing Office), 949-961.

Manuscrito recibido: Agosto 9, 2004

Manuscrito corregido recibido: Abril 30, 2005

Manuscrito acceptado: Mayo 3, 2005 